\title{
Improved Initial Synchronisation in the Presence of Frequency Offset in UMTS FDD Mode
}

\author{
Valentina Lomi $^{1}$, Gianfranco L. Pierobon ${ }^{2}$, Daniele Tonetto ${ }^{1}$, and \\ Lorenzo Vangelista ${ }^{1}$ \\ 1 Telit Mobile Terminals S.p.A., \\ via Masini 2, 35129 Padova, Italy \\ \{valentina.lomi, daniele.tonetto, lorenzo.vangelista\}@telital.com \\ ${ }^{2}$ Università di Padova, Dipartimento di Elettronica e Informatica, \\ via Gradenigo 6/A, 35131 Padova, Italy \\ gianfranco.pierobon@unipd.it
}

\begin{abstract}
The UMTS-FDD system, one of the members of the ITU IMT-2000 third generation standard for terrestrial cellular systems, employs pruned Golay sequences to enable initial synchronisation of the mobile terminals to the network. In this paper a low complexity solution for initial synchronisation is proposed, which is able to counteract the performance degradation introduced by large frequency offsets occurring in the mobile station receiver. Simulation results are provided to validate the proposed solution.
\end{abstract}

Keywords: cell search, UMTS, FDD, Golay sequences, synchronisation

\section{Introduction}

The initial synchronisation is the process of a mobile station in a cellular CDMA network getting synchronised in time to the (strongest) base station and acquiring the scrambling code that base station uses for the downlink traffic channels.

To let the mobile get synchronised to the network, the UMTS-FDD system [1] [2] provides two "bursty" pilot channels ("primary" and "secondary" synchronisation channels) and a continuous pilot channel. In this paper we focus on the "primary" channel which is based on the repetition of a non-scrambled Golay sequence common to all cells and which is needed to perform slot synchronisation (the first step of the initial synchronisation procedure, see [3]).

During the standardisation process particular attention has been paid to the necessity of an implementation with requirements of low complexity and of robustness to the frequency offsets, as low cost mobile stations in UMTS systems may have large initial frequency offsets, up to $26 \mathrm{kHz}$ [4]. In [4] [5] and [6], algorithms which are able to counteract the effect of the frequency offset on the initial synchronisation procedure are presented.

In this paper we propose another solution for slot synchronisation in the presence of a frequency offset, which performs better than [4] in most cases. The theoretical basis for our solution is a theorem, proven in this paper, according

E. Gregori et al. (Eds.): NETWORKING 2002, LNCS 2345, pp. 1165-1171 2002.

(C) Springer-Verlag Berlin Heidelberg 2002 
to which the Golay sequences rotated at the receiver by a frequency offset still preserve the "Golay property".

The paper is organised as follows. Section 2 describes the slot synchronisation procedure in the UMTS-FDD system. Section 3 demonstrates the previously mentioned theorem. Section 4 describes the proposed algorithm and its performance. Conclusions are drawn in Section 5

\section{$2 \quad$ System Model}

For purposes of the slot synchronisation (see [1] for full details), we model the baseband representation of the signal received and sampled at the mobile station at chip rate $1 / T_{c}, T_{c}=1 / 3840000 \mathrm{~s}$, as

$$
x_{r}\left(k T_{c}\right)=A_{c h} \cdot e^{\jmath\left(2 \pi \Delta f k T_{c}+\theta\right)} \cdot p_{S C H}\left(\left(\left(k-k_{0}\right) \bmod Q\right) T_{c}\right)+\tilde{w}\left(k T_{c}\right)
$$

where

$-Q=2560 ; Q T_{c}$ is the time interval called slot in the UMTS specifications;

- $A_{c h}$ is a real value modelling a constant channel attenuation;

$-\Delta f$ is the receiver frequency offset and $\theta$ is an unknown phase;

$-k_{0} T_{c}$ is the time offset (unknown to the receiver): its estimation is actually the target of slot synchronisation;

- $\tilde{w}\left(k T_{c}\right)$ is white Gaussian noise with variance $\sigma^{2}$;

- $p_{S C H}\left((k \bmod Q) T_{c}\right)$ is the primary synchronisation channel, common to all UMTS base stations. The sequence

$$
p_{S C H}\left(k T_{c}\right)= \begin{cases}\frac{1}{\sqrt{2}} \frac{1}{\sqrt{256}}(1+\mathrm{j}) a(k) & \text { for } 0 \leq k<256 \\ 0 & \text { for } 256 \leq k<Q\end{cases}
$$

repeats every slot. $a(k)$ is a pruned Golay complementary sequence generated as follows:

$$
\begin{aligned}
a_{0}(k) & =\delta(k) \quad b_{0}(k)=\delta(k) \\
a_{n}(k) & =a_{n-1}(k)+W_{n} \cdot b_{n-1}\left(k-D_{n}\right) \\
b_{n}(k) & = \begin{cases}a_{n-1}(k)-W_{n} \cdot b_{n-1}\left(k-D_{n}\right) & n=1,2,3,5,7,8 \\
a_{n}(k) & n=4,6\end{cases} \\
a(k) & =a_{8}(k)
\end{aligned}
$$

with $k=0,1,2, \ldots, 255$, where

$$
\begin{aligned}
{\left[D_{1}, D_{2}, D_{3}, D_{4}, D_{5}, D_{6}, D_{7}, D_{8}\right] } & =[128,64,16,32,8,1,4,2] \\
{\left[W_{1}, W_{2}, W_{3}, W_{4}, W_{5}, W_{6}, W_{7}, W_{8}\right] } & =[1,-1,1,1,1,1,1,1]
\end{aligned}
$$

Let $C N R$ be the ratio between the power of the primary synchronisation channel and the power of noise, hence $C N R=A_{c h}^{2} /\left(256 \sigma^{2}\right)$.

An efficient estimation of $k_{0}$ can be obtained with the Budisin correlator (see [7]) shown in Fig[1, applied with the following procedure: 


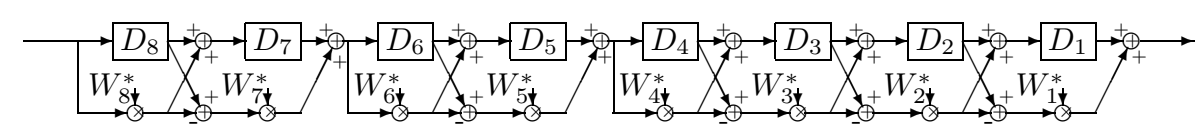

Fig. 1. Budisin correlator

1. letting $L$ be the number of slots in a synchronisation time, calculate

$$
m(n)=\sum_{\ell=0}^{L-1}\left|c\left((n+\ell Q) T_{c}\right)\right| \quad n=0,1, \ldots, Q-1
$$

where $c\left(k T_{c}\right)$ is the signal at the output of the correlator;

2. find $n_{0}$ such that $m\left(n_{0}\right) \geq m(n), n=0,1, \ldots, Q-1$;

3. calculate $\hat{k_{0}}=n_{0}-255$.

\section{Rotated Golay Sequences}

Theorem 1. If the sequence $a(k)$ is a pruned Golay complementary sequence defined by the recursive equations (3), (4), (5) and (6), then the sequence $\hat{a}(k)=a(k) e^{j 2 \pi \Delta f k T_{c}}$ is a pruned Golay complementary sequence too (from now on called rotated Golay sequence), which can be obtained from the recursive equations (3), (4), (5), and (6) with the substitution $W_{n} \mapsto \hat{W}_{n}=W_{n} \cdot e^{j 2 \pi \Delta f D_{n} T_{c}}$.

Proof. Equations (3), (4), (5) and (6) can be rewritten in the $z$-transform domain as

$$
\begin{aligned}
A_{n}(z) & =A_{n-1}(z)+W_{n} z^{-D_{n}} B_{n-1}(z) \\
B_{n}(z) & = \begin{cases}A_{n-1}(z)-W_{n} z^{-D_{n}} B_{n-1}(z) & \text { for } n=1,2,3,5,7,8 \\
A_{n}(z) & \text { for } n=4,6\end{cases} \\
A(z) & =A_{8}(z)
\end{aligned}
$$

with the initial condition $A_{0}(z)=B_{0}(z)=1$. Defining

$$
\begin{gathered}
\hat{a}_{n}(k)=a_{n}(k) e^{\jmath 2 \pi \Delta f T_{c}} \\
\hat{b}_{n}(k)=b_{n}(k) e^{\jmath 2 \pi \Delta f T_{c}}
\end{gathered}
$$

and substituting $z \mapsto z e^{-\jmath 2 \pi \Delta f T_{c}}$ in (9), (10) and (11), we have that the sequence $\hat{a}(k)$ can be represented, in the $z$-transform domain, by the recursive equations

$$
\begin{aligned}
& \hat{A}_{n}(z)=\hat{A}_{n-1}(z)+W_{n} e^{\jmath 2 \pi D_{n} \Delta f T_{c}} z^{-D_{n}} \hat{B}_{n-1}(z) \\
& \hat{B}_{n}(z)= \begin{cases}\hat{A}_{n-1}(z)-W_{n} e^{\jmath 2 \pi D_{n} \Delta f T_{c}} z^{-D_{n}} \hat{B}_{n-1}(z) & \text { for } n=1,2,3,5,7,8 \\
\hat{A}_{n}(z) & \text { for } n=4,6\end{cases} \\
& \hat{A}(z)=\hat{A}_{8}(z)
\end{aligned}
$$

with the initial conditions $\hat{A}_{0}(z)=\hat{B}_{0}(z)=1$. 


\section{The Proposed Synchronisation Algorithm and Its Performances}

It is known that the usual synchronisation procedure, described in Section 2, is very sensitive to frequency offsets. It can be shown (see [4]) that the signal-tonoise ratio degradation in the output of the correlator matched to the sequence $a(k)$ is proportional to

$$
\frac{\sin ^{2} N \pi \Delta f T_{c}}{N \sin ^{2} \pi \Delta f T_{c}}
$$

with $N=256$. Hence the correlation peak vanishes at all when $\Delta f=\Delta f_{ \pm}=$ $\pm 1 / N T_{c}= \pm 15 \mathrm{kHz}$.

This consideration together with Theorem 11 lead us to propose the new solution, depicted in Fig. 2, which uses three Budisin correlators, one matched to the sequence $a(k)$, one matched to $a(k) \cdot e^{j 2 \pi \Delta f_{+} k T_{c}}$ and one matched to $a(k) \cdot e^{\jmath 2 \pi \Delta f_{-} k T_{c}}$. 1 The $M A X$ block takes the sequences applied at its input, determines the maximum in the set of all the values assumed by the sequences and produces as an output the input sequence to which the maximum belongs.

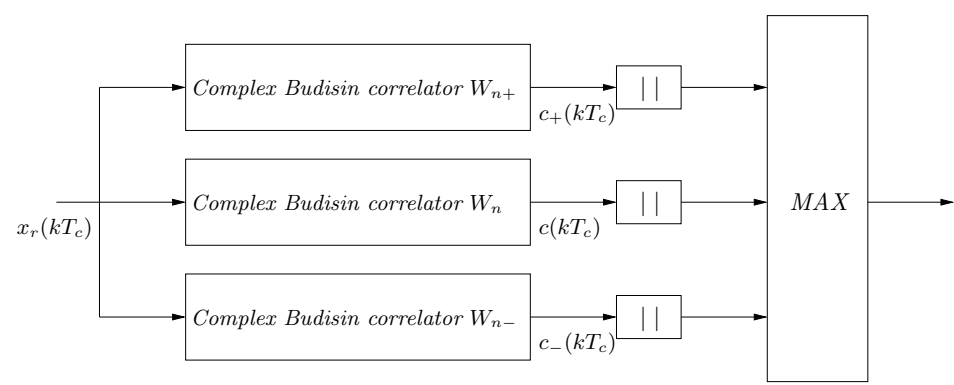

Fig. 2. The proposed algorithm

Unfortunately the proposed synchronisation scheme has a higher implementation complexity than usual schemes because the coefficients $W_{n \pm}$ are $e^{\jmath 2 \pi \Delta f_{ \pm} D_{n} T_{c}}$ instead of simply \pm 1 and three correlators, instead of only one, are used.

In order to reduce the implementation complexity we make the following approximations for the coefficients of the upper and lower correlators:

$$
\begin{aligned}
& W_{m+} \approx W_{m} \\
& W_{m-} \approx W_{m}
\end{aligned} \quad \text { for } m=3,4,5,6,7,8
$$

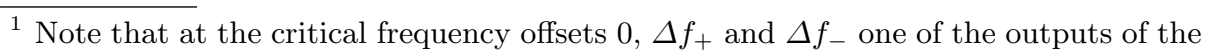
three correlators raises its maximum, while the others have there their minima. 


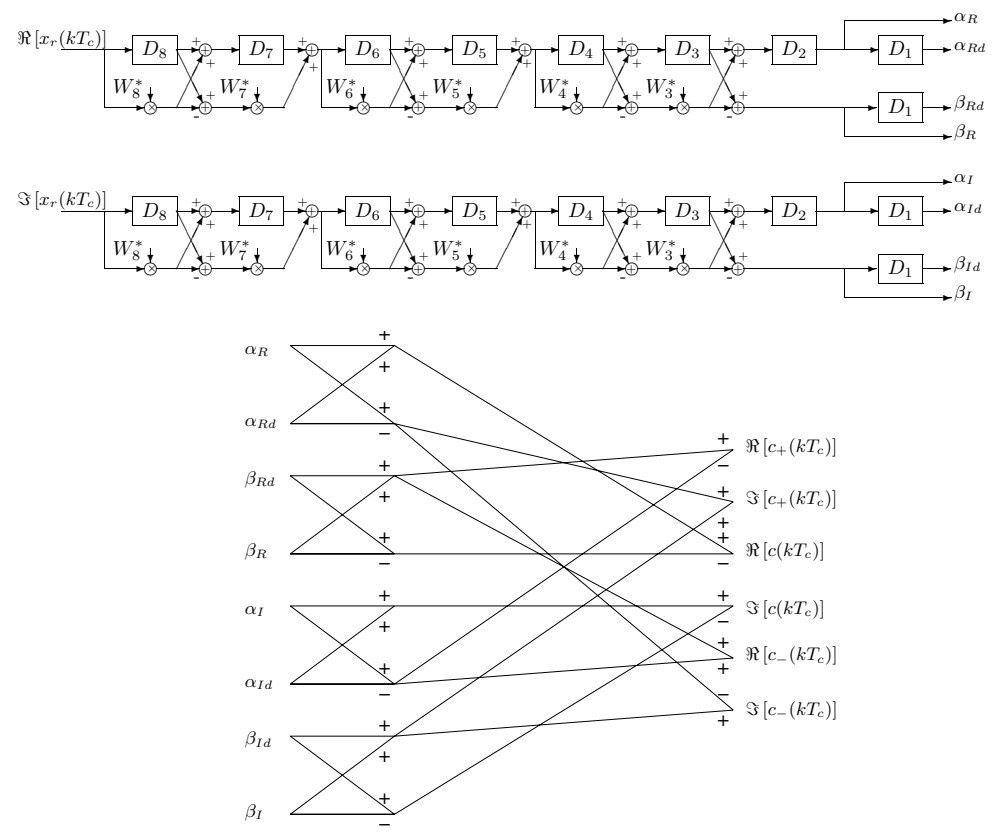

Fig. 3. Reduced complexity scheme

It can be demonstrated that these approximations have the correlators matched to sequences rotated by a staircase phase instead of a linear increasing phase. Taking into account the above assumptions, computations depicted in Fig. 2 can be re-organised as shown in Fig. 3 in a reduced complexity scheme. Note that, while the single Budisin correlator (considering both in-phase and quadrature components) needs 26 sums per output, the computations required by the proposed algorithm in this simplified implementation are 34 sums per output.

The performance of the proposed algorithm in a flat fading channel with $9.26 \mathrm{~Hz}$ Doppler is shown in Fig. 4 with no frequency offset and with a $20 \mathrm{kHz}$ frequency offset. In both cases it is compared with the performances obtained with a single Budisin correlator and with the algorithm described in 4 . (indicated with the label "Wang-Ottosson"). The algorithm proposed in this paper shows a very good performance both at $0 \mathrm{kHz}$ and $20 \mathrm{kHz}$. The synchronisation error is also plotted versus the frequency offset in Fig. 5 for both the algorithm in [4] (indicated with the label "W-O") and the proposed method. Examined CNR values are $-21 \mathrm{~dB}$ and $-13 \mathrm{~dB}$. The proposed algorithm shows the best behaviour for all the examined frequency range, except for the values around $\Delta f_{+} / 2$. 
(a)

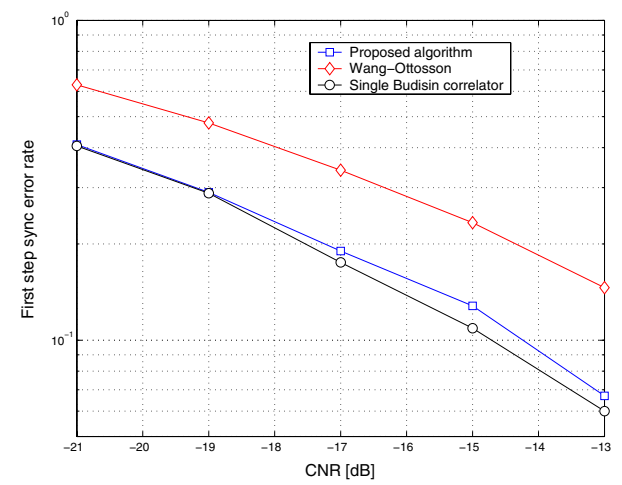

(b)

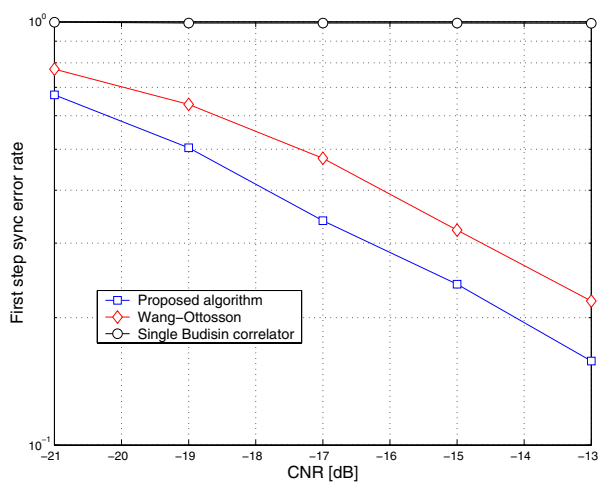

Fig. 4. First step performance with a 0 (a) and $20 \mathrm{kHz}$ (b) frequency offset $(L=15)$

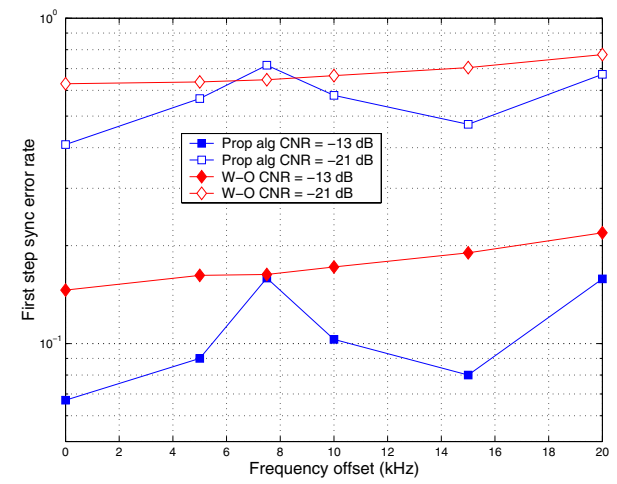

Fig. 5. First step performance in two different CNR conditions $(L=15)$

\section{Conclusions}

We have presented an innovative method for the first step of the UMTS-FDD initial synchronisation procedure which is able to counteract the degrading effect of the frequency offset. The algorithm has a low complexity implementation. Simulations indicate that it can offer a good performance for all the CNR and frequency offset values of interest.

\section{References}

1. 3GPP 3G TS 25.211 "Physical channels and mapping of physical channels onto transport channels (FDD)", version 4.1.0 June 2001.

2. 3GPP 3G TS 25.213 "Spreading and modulation (FDD)", version 4.1.0 June 2001.

3. 3GPP 3G TS 25.214 "Physical layer procedures (FDD)", version 4.1.0 June 2001. 
4. Y.-P. E. Wang, T. Ottosson, "Cell search in W-CDMA", IEEE Journal on Selected Areas in Communications, Vol. 18, No. 8, Aug. 2000.

5. K.-M. Lee, J.-Y. Chun, "An initial cell search scheme robut to frequency error in W-CDMA system", PIMRC 2000, Vol. 2, 2000.

6. S.-Y. Hwang, B.-J. Kang, J.-S. Kim, "Performance analysis of initial cell search using time tracker for W-CDMA", GLOBECOM 2001, Vol. 5, 2001.

7. S. Z. Budisin, "Efficient pulse compressor for Golay complementary sequences", Electronics Letters, Vol. 27, No. 3, Jan. 1991. 